\title{
Effects of nonlocal dispersive interactions on self-trapping excitations
}

\author{
Gaididei, Yu.B.; Mingaleev, S.F.; Christiansen, Peter Leth; Rasmussen, Kim
}

Published in:

Physical Review E. Statistical, Nonlinear, and Soft Matter Physics

Link to article, DOI:

10.1103/PhysRevE.55.6141

Publication date:

1997

Document Version

Publisher's PDF, also known as Version of record

Link back to DTU Orbit

Citation (APA):

Gaididei, Y. B., Mingaleev, S. F., Christiansen, P. L., \& Rasmussen, K. (1997). Effects of nonlocal dispersive interactions on self-trapping excitations. Physical Review E. Statistical, Nonlinear, and Soft Matter Physics, 55(5), 6141-6149. https://doi.org/10.1103/PhysRevE.55.6141

\section{General rights}

Copyright and moral rights for the publications made accessible in the public portal are retained by the authors and/or other copyright owners and it is a condition of accessing publications that users recognise and abide by the legal requirements associated with these rights.

- Users may download and print one copy of any publication from the public portal for the purpose of private study or research.

- You may not further distribute the material or use it for any profit-making activity or commercial gain

- You may freely distribute the URL identifying the publication in the public portal 


\title{
Effects of nonlocal dispersive interactions on self-trapping excitations
}

\author{
Yu. B. Gaididei and S. F. Mingaleev \\ Bogolyubov Institute for Theoretical Physics, 252143 Kiev, Ukraine \\ P. L. Christiansen and K. Ф. Rasmussen \\ Department of Mathematical Modelling, The Technical University of Denmark, DK-2800 Lyngby, Denmark
}

(Received 7 June 1996; revised manuscript received 24 October 1996)

\begin{abstract}
A one-dimensional discrete nonlinear Schrödinger (NLS) model with the power dependence $r^{-s}$ on the distance $r$ of the dispersive interactions is proposed. The stationary states $\psi_{n}$ of the system are studied both analytically and numerically. Two types of stationary states are investigated: on-site and intersite states. It is shown that for $s$ sufficiently large all features of the model are qualitatively the same as in the NLS model with a nearest-neighbor interaction. For $s$ less than some critical value $s_{\mathrm{cr}}$, there is an interval of bistability where two stable stationary states exist at each excitation number $N=\Sigma_{n}\left|\psi_{n}\right|^{2}$. For cubic nonlinearity the bistability of on-site solitons may occur for dipole-dipole dispersive interaction $(s=3)$, while $s_{\mathrm{cr}}$ for intersite solitons is close to 2.1. For increasing degree of nonlinearity $\sigma, s_{\mathrm{cr}}$ increases. The long-distance behavior of the intrinsically localized states depends on $s$. For $s>3$ their tails are exponential, while for $2<s<3$ they are algebraic. In the continuum limit the model is described by a nonlocal NLS equation for which the stability criterion for the ground state is shown to be $s<\sigma+1$. [S1063-651X(97)12205-X]

PACS number(s): 03.40.Kf, 42.65.Tg, 63.20.Pw, 87.10.+e
\end{abstract}

\section{INTRODUCTION}

Recently, the determination of the dynamical properties of physical systems with competition between discreteness, nonlinearity, and dispersion has attracted a growing interest because of their wide applicability in various physical problems. Examples are coupled optical fibers, arrays of coupled Josephson junctions, nonlinear charge and excitation transport in biological macromolecules, elastic energy transfer in anharmonic chains, and charge transport in hydrogen-bonded systems. It is well known that the balance between nonlinearity and dispersion in a weak nonlinearity (large dispersion) limit provides the existence of low-energy solitonlike excitations. They are very robust and propagate without energy loss, and their collisions are almost elastic. Due to their robust character the soliton excitations are important in the coherent excitation transport in biological macromolecules $[1,2]$ and charge transport in organic semiconductors $[3,4]$.

As a result of the interplay between discreteness, dispersion, and nonlinear interactions, new nonlinear excitations, namely, intrinsically localized oscillatory states, may appear. The properties of the localized modes have been intensively studied during the past years [5-14]. For monatomic lattices with a nearest-neighbor harmonic interaction and quartic anharmonic interaction the localized states were found [9-11] to have frequencies lying above the phonon band. In the case of a one-dimensional (1D) nonlinear Schrödinger (NLS) lattice [15] a localized mode lying below the linear excitation band in the small-amplitude limit reduces to the one-soliton solution of the continuum NLS equation.

Recently, a discrete NLS equation with "tunable" diagonal and off-diagonal nonlinearities that includes the integrable Ablowitz-Ladik system [5] as a limit was introduced in $[16,17]$. It was shown that reflection and translational symmetries of the integrable NLS are broken by diagonal nonlinearity and the properties of the Peierls-Nabarro poten- tial as a function of the tuning parameter were studied. A further study of the stationary properties of these systems was recently performed in [18]. The nonintegrable dynamics of a 1D discrete NLS system with an arbitrary degree of nonlinearity was investigated in [19]. An analytical stability criterion for solitons in the discrete NLS equation with an arbitrary degree of nonlinearity was obtained in Ref. [20]. It was shown that a process of quasicollapse may take place when an unstable soliton transfers into an intrinsically localized mode.

In the main part of the previous studies the dispersive interaction was assumed to be short ranged and a nearestneighbor approximation was used. However, there exist physical situations that definitely cannot be described in the framework of this approximation. The excitation transfer in molecular crystals [21] and the vibron energy transport in biopolymers [2] are due to the transition dipole-dipole interaction with a $1 / r^{3}$ dependence on the distance $r$. The DNA molecule contains charged groups, with a long-range Coulomb interaction $(1 / r)$ between them. In systems where the dispersion curves of two elementary excitations are close or intersect, effective long-range transfer occurs. Such a situation arises for excitons and photons in semiconductors and molecular crystals (so-called polariton effects [21]).

Until recently there have been few theoretical and numerical studies of the effect of long-range interactions (LRI's) on the properties of nonlinear excitations. Nonlinear waves in a one-dimensional (1D) chain with a Lennard-Jones $(2 n, n)$ interatomic potential were studied in [22]. It was shown that the dynamics is governed by the Benjamin-Ono equation in the case $n=2$ or by the Korteweg-de Vries equation for $n \geqslant 4$. The effective mass of solitons in the FrenkelKontorova model with a repulsive LRI, their shapes and Peierls barriers were investigated in Ref. [23]. In [24] an implicit form of solitons was obtained in a sine-Gordon system with a LRI of the Kac-Baker type [25,26] and the de- 
pendence of the soliton width and energy on the radius of the LRI was analyzed. It was postulated in [27] that the nonlinear term in the sine-Gordon equation has a nonlocal character and different soliton states, of topological charge zero, were found to exist at a large enough radius of the interaction. In [28] the effects of a long-range harmonic interaction in a chain with short-range anharmonicity were considered. It was demonstrated that the existence of two velocitydependent competing length scales leads to two types of solitons with characteristically different width and shapes for two velocity regions separated by a gap. The effects of longrange interactions of the Kac-Baker type were studied in static and dynamic nonlinear Klein-Gordon [23,29,30] and nonlinear Schrödinger [31] continuum models. In [31] we proposed a nonlocal NLS equation for systems with longrange dispersion effects. In contrast to the usual NLS equation, stationary solutions exist only for a finite interval of the excitation number. In the upper part of this interval two different kinds of stationary solutions were found. The kind that contains a cusp soliton was shown to be unstable. It was also pointed out that moving solitons radiate with a wavelength proportional to the velocity. In Ref. [32] we proposed a nonlocal discrete NLS model where the dispersive interaction had a power dependence on the distance. It was found that there is an interval of bistability in the NLS models with a long-range dispersive interaction. One of these states is a continuumlike soliton and the other is an intrinsically localized mode.

The goal of this paper is to investigate both types of soliton states in the discrete NLS model, on-site and intersite states, and study the motion of solitons and Peierls-Nabarro pinning. We develop also a quasicontinuum approach to the problem. In Sec. II we present the analytical theory and the results of numerical simulations of stationary states of the discrete NLS model with a long-range dispersive interaction. We discuss the bistability phenomenon for the two types (on-site and intersite) of soliton solutions and their stability. In the analytical part of this section we use a variational approach exploiting an exponential-like function as a trial function. Then, in Sec. III we investigate the long-distance behavior of the nonlinear excitations and show that intrinsically localized states of the discrete NLS model with a dispersive interaction decaying slower than $1 / r^{3}$ have algebraic tails. In Sec. IV we investigate the soliton states in a quasicontinuum approximation and derive a continuum nonlocal NLS equation. We show that the degree of nonlocality depends on the dispersion parameter $s$. In particular, for $s=2$ (inverse square dependence on the distance of excitation transfer) we obtain an equation, which we denote the Hilbert-NLS equation, that has a form that is closely related to the Benjamin-Ono equation. The stability of the ground state of the nonlocal NLS equation is studied. Section V presents the concluding discussion.

\section{SYSTEM AND EQUATIONS OF MOTION}

The model we study is described by the Hamiltonian

$$
H=T+U_{\sigma},
$$

where

$$
T=\frac{1}{2} \sum_{n, m(n \neq m)} J_{n-m}\left|\psi_{m}-\psi_{n}\right|^{2}
$$

is the dispersive energy of the excitation and

$$
U_{\sigma}=-\frac{1}{(\sigma+1)} \sum_{n}\left|\psi_{n}\right|^{2(\sigma+1)}
$$

is the potential energy that describes a self-interaction of the quasiparticle. In Eqs. (1)-(3) $n$ and $m$ are site indices and $\psi_{n}$ is the excitation wave function. We investigate the model with the following power dependence on the distance of the matrix element of excitation transfer $J_{n-m}=J /|n-m|^{s}$. The constant $J$ characterizes the strength of the transfer and $s$ is a parameter that is introduced to cover different physical situations from the nearest-neighbor approximation $(s=\infty)$ and the dipole-dipole interaction $(s=3)$ to the long-range Coulomb interaction $s=1$. We shall show that this equation having tunable properties illuminates both the competition between nonlinearity and dispersion and the interplay of longrange interactions and lattice discreteness. The degree of nonlinearity $\sigma$ is another parameter that we include for generality, as it has been done in several previous works $[19,20,33,34]$; however, the main part of this paper is concerned with the cubic $(\sigma=1)$ case.

From the Hamiltonian (1) we obtain the equation of motion $i \dot{\psi}_{n}=\partial H / \partial \psi_{n}^{*}$ for the excitation wave function in the form

$$
i \dot{\psi}_{n}+\sum_{m(m \neq n)} J_{n-m}\left(\psi_{m}-\psi_{n}\right)+\left|\psi_{n}\right|^{2 \sigma} \psi_{n}=0
$$

where the overdot denotes the time derivative. The Hamiltonian $H$ and the number of excitations

$$
N=\sum_{n}\left|\psi_{n}\right|^{2}
$$

are conserved quantities. Obviously, the Lagrangian for Eq. (4) can be written via the Legendre transform of $H$ as

$$
L=i \sum_{n} \frac{1}{2}\left(\dot{\psi}_{n} \psi_{n}^{*}-\dot{\psi}_{n}^{*} \psi_{n}\right)-H
$$

We are interested in stationary solutions of Eq. (4) of the form

$$
\psi_{n}=\phi_{n} \exp (i \Lambda t)
$$

with a real shape function $\phi_{n}$ and a frequency $\Lambda$ (there seems to be a rather great number of names for this parameter; we have here chosen the term "frequency" since this appears to be the most common; see, e.g., [34]). This reduces the Lagrangian (6) to $L=\Lambda \Sigma_{n} \phi_{n}^{2}-H$ and the determining equation for the functions $\phi_{n}$ becomes

$$
\Lambda \phi_{n}=J \sum_{m(m \neq n)}|n-m|^{-s}\left(\phi_{m}-\phi_{n}\right)+\phi_{n}^{(2 \sigma+1)} .
$$

Thus Eq. (8) is the Euler-Lagrange equation for the problem of minimizing $H$ under the constraint $N=$ const. 
To obtain an approximate solution of the problem we use an ansatz for a localized state in the form

$$
\phi_{n}=\sqrt{\frac{N \sinh \alpha}{\cosh \{\alpha(2 \delta-1)\}}} \exp (-\alpha|n-\delta|),
$$

where $\alpha$ is a trial parameter and $\delta$ is the position of the center of the localized state, which, without loss of generality, can be restricted to $0 \leqslant \delta<1$ for the infinite chain. The ansatz (9) is chosen to automatically satisfy the normalization condition

$$
\sum_{n} \phi_{n}^{2}=N
$$

such that the problem of minimizing $H$ under the constraint $N=$ const is reduced to the problem of satisfying the equation $d H / d \alpha=0$.

Variational approaches similar to the one described above have recently $[35,34]$ been used for the nearest-neighbor case of Eq. (4) to analyze static and dynamical properties of the solitions. In particular, the work of Malomed and Weinstein [34] is based on the same ansatz, while the work of Aceves et al. [35] is based on an essentially different ansatz that is too complicated for analytical analysis.

It will be shown in Sec. III that the long-distance behavior of the excitation wave function $\phi_{n}$ drastically depends on the value of the dispersive parameter $s$. Only for $s>3$ are the tails of $\phi_{n}$ exponential. However, a qualitative description of the energy spectrum of the system can be obtained using the trial function in the form (9) at any $s$.

To calculate the kinetic energy $T$ we use the discrete Fourier transform

$$
\Phi(k)=\sum_{n} \exp (i k n) \phi_{n}, \quad J(k)=\sum_{n} \exp (i k n) J_{n},
$$

which permits us to rewrite Eq. (2) in the form

$$
T=\frac{1}{M} \sum_{k} \mathcal{L}(k)|\Phi(k)|^{2}=\frac{1}{2 \pi} \int_{-\pi}^{\pi} \mathcal{L}(k)|\Phi(k)|^{2},
$$

where the spectrum function

$$
\mathcal{L}(k)=J(0)-J(k)
$$

determines the linear dispersion of the excitations and $M$ is the number of sites in the system $(M \rightarrow \infty)$. For convenience we shall use

$$
J=\frac{1}{\zeta(s)}
$$

with $\zeta(s)$ being Riemann's zeta function

$$
\zeta(s)=\sum_{n=1}^{\infty} n^{-s}
$$

to have $J(0)$ independent of $s$. Using

$$
\begin{aligned}
J(k) & =2 J \sum_{n=1}^{\infty} n^{-s} \cos k n=\frac{2}{\zeta(s)} \operatorname{Re}\left\{F\left(e^{i k}, s\right)\right\} \\
& =\frac{2 J}{\Gamma(s)} \int_{0}^{1} d z \frac{(\cos k-z)[\ln (1 / z)]^{s-1}}{z^{2}-2 z \cos k+1},
\end{aligned}
$$

where

$$
F(z, s)=\sum_{n=1}^{\infty}\left(z^{n} / n^{s}\right)
$$

is the so-called Jonqière function (its properties are described in Ref. [36]) and

$$
\begin{aligned}
|\Phi(k)|^{2}= & N \frac{\sinh \alpha}{\cosh [\alpha(2 \delta-1)]}\left(\frac{\sinh ^{2} \alpha}{(\cosh \alpha-\cos k)^{2}}\right. \\
& \left.+2 \frac{\sinh (\alpha \delta) \sinh [\alpha(\delta-1)]}{\cosh \alpha-\cos k}\right),
\end{aligned}
$$

we obtain, for the kinetic energy,

$$
T=2 N\left\{1-J\left(\frac{F\left(e^{-\alpha}, s-1\right) \sinh \alpha}{\cosh [\alpha(2 \delta-1)]}+F\left(e^{-\alpha}, s\right)\right)\right\} .
$$

Inserting Eq. (9) into Eq. (3) we get

$$
U_{\sigma}=-\frac{N^{\sigma+1}}{\sigma+1} f_{\sigma}
$$

where

$$
f_{\sigma}=\left(\frac{\sinh ^{\sigma+1} \alpha}{\cosh ^{\sigma+1}[\alpha(2 \delta-1)]} \frac{\cosh [(\sigma+1) \alpha(2 \delta-1)]}{\sinh [(\sigma+1) \alpha]}\right) .
$$

According to the variational principle, we should satisfy the equation $d H / d \alpha=0$, which yields

$$
\begin{aligned}
N= & 8^{\sigma+1} J\left\{\left(1-\frac{\cosh \alpha}{\cosh [\alpha(2 \delta-1)]}+(2 \delta-1)\right.\right. \\
& \left.\times \frac{\sinh \alpha \tanh [\alpha(2 \delta-1)]}{\cosh [\alpha(2 \delta-1)]}\right) F\left(e^{-\alpha}, s-1\right) \\
& \left.+\frac{\sinh \alpha}{\cosh ^{2}[\alpha(2 \delta-1)]} F\left(e^{-\alpha}, s-2\right)\right\}\left(\frac{d f_{\sigma}}{d \alpha}\right)^{-1} .
\end{aligned}
$$

As a direct consequence of Eq. (8), the frequency $\Lambda$ can be obtained as

$$
\Lambda=-\frac{1}{N}\left(T+2 U_{\sigma}\right)
$$

with $T$ and $U_{\sigma}$ being defined by Eqs. (19) and (20). We shall study in detail the stationary states of the system for the case $\sigma=1$. The two types of stationary states, on-site $(\delta=0)$ and intersite $(\delta=1 / 2)$ states, will be considered separately. 


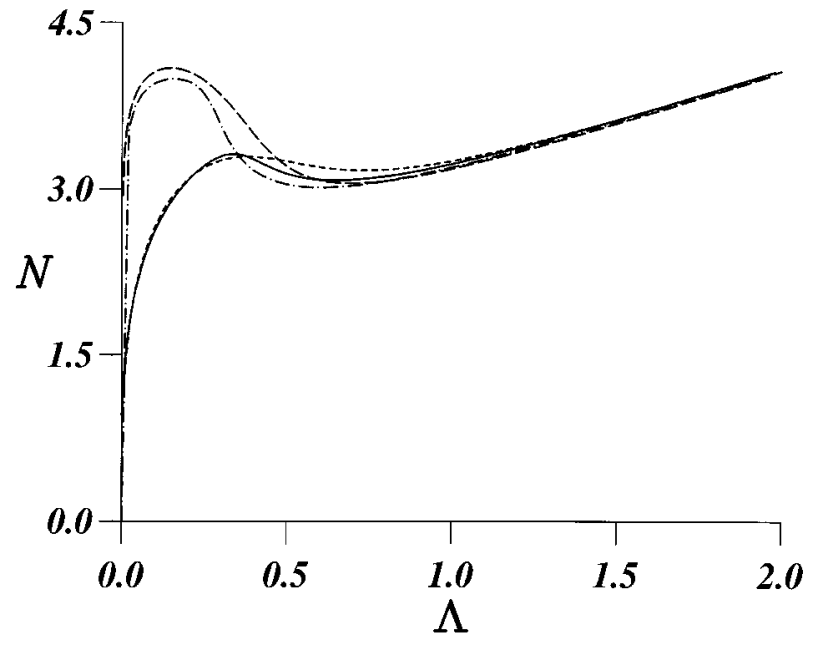

FIG. 1. Number of excitations $N$ versus frequency $\Lambda$. A comparison between analytical dependence Eq. (21) for $s=2.1$ (longdashed line), 2.5 (short-dashed line), and numerical dependence from Eq. (4) for $s=2.1$ (dash-dotted line) and 2.5 (full line).

\section{A. On-site localized states: $\delta=0$}

Figures 1 and 2 show the dependence $N(\Lambda)$ obtained analytically from Eqs. (22) and (23) for $\sigma=1$ and from direct numerical solution of Eq. (8). A monotonic dependence is obtained for $s>s_{\mathrm{cr}}$. For $s_{\mathrm{cr}}>s>2$ the dependence becomes nonmonotonic (of $\mathcal{N}$ type) with a local maximum and a local minimum. These extrema coalesce at $s=s_{\mathrm{cr}} \simeq 2.72$ [from Eqs. (22) and (23)] and $s=s_{\mathrm{cr}} \simeq 3.03$ [from the numerical solution of Eq. (8)]. For $s<2$ the local maximum disappears. Thus the main features of all discrete NLS models with dispersive interaction $J_{n-m}$ decreasing faster than $|n-m|^{-s_{\text {cr }}}$ coincide qualitatively with the features obtained in the nearest-neighbor approximation where only one on-site stationary state exists for any excitation number. However, in the case of the long-range nonlocal NLS equation (4), i.e., $2<s<s_{\mathrm{cr}}$, there exist for each $N$ in the interval

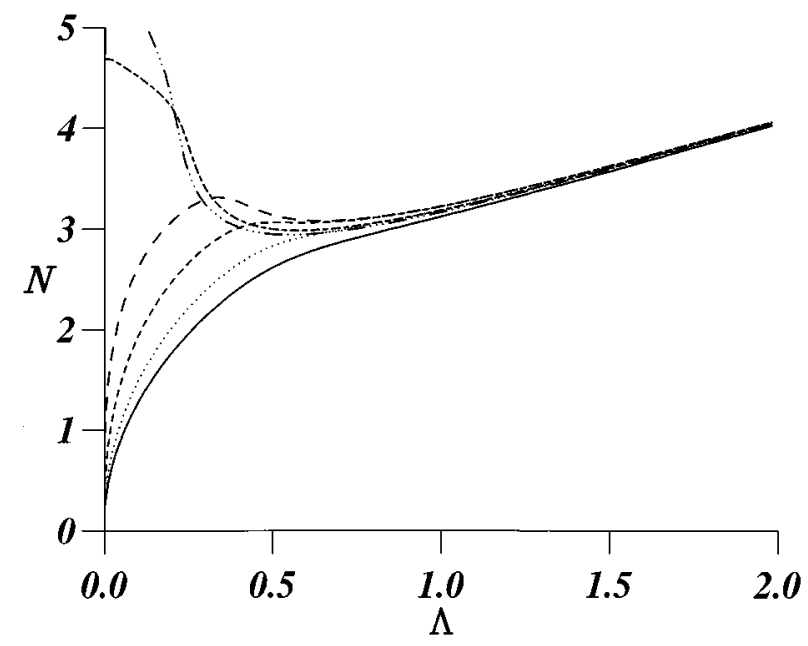

FIG. 2. Number of excitations $N$ versus frequency $\Lambda$ numerically from Eq. (7) for $s=\infty$ (full line), 4 (dotted line), 3 (shortdashed line), 2.5 (long-dashed line), 2 (short-dash-long-dashed line), 1.9 (dash-dotted line).

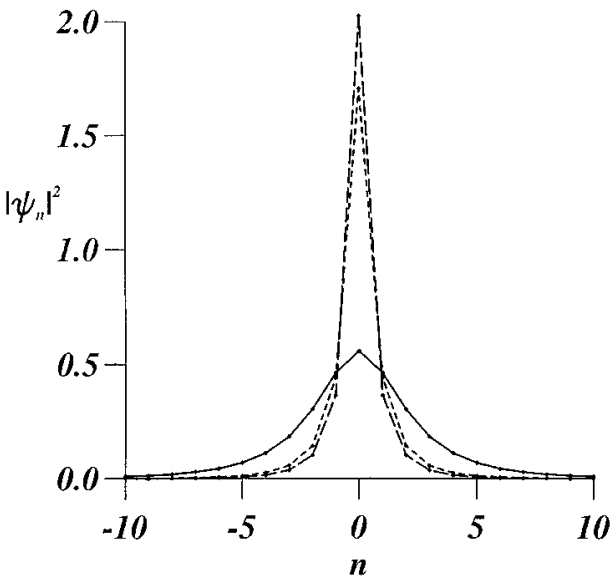

FIG. 3. Shapes of the three stationary states for $s=2.5$ and $N=3.1$ : the stable states $\Lambda=0.21$ (long-dashed line) and $\Lambda=0.74$ (full line) and the ustable state $\Lambda=0.57$ (short-dashed line).

$\left[N_{l}(s), N_{u}(s)\right]$ three stationary states with frequencies $\Lambda_{1}(N)<\Lambda_{2}(N)<\Lambda_{3}(N)$. In particular, this means that in the case of the dipole-dipole interaction $(s=3)$ multiple solutions exist. The observed bistability is very similar to the bistability observed in Refs. [20] and [34], where the nearestneighbor case with an arbitrary degree of nonlinearity $\sigma$ was studied. The bistability appears in this case for $\sigma$ above a certain critical value.

Figure 3 shows that the shapes of these solutions differ significantly. The low-frequency states are wide and continuumlike, while the high-frequency solutions represent intrinsically localized states with a width of a few lattice spacings. For $s \gtrsim 2$ we can expand Eq. (22) in the limits $\alpha \rightarrow 0$ and $1 / \alpha \rightarrow 0$ and obtain that the inverse widths of these two stable states are

$$
\alpha_{1} \approx\left(\frac{N}{8 J}\right)^{1 /(s-2)}=\left(\frac{N}{8 J}\right)^{\ln l /(1-2 \ln l)}, \quad \alpha_{3} \approx \ln \left(\frac{N}{J}\right),
$$

where $l=\exp (1 / s)$ is the characteristic length scale of the dispersive interaction. It is seen from these expressions that the existence of two so different soliton states for one value of the excitation number $N$ is due to the presence of two different length scales in the system: the usual scale of the NLS model, which is related to the competition between nonlinearity and dispersion (expressed in terms of the ratio $N / J$ ), and the range of the dispersive interaction $l$.

Exploiting that, for $\alpha<\pi$, the Jonqière function $F\left(e^{-\alpha}, s\right)$ can be represented in the form

$$
F\left(e^{-\alpha}, s\right)=\Gamma(1-s) \alpha^{s-1}+\sum_{r=0}^{\infty} \zeta(s-r) \frac{(-\alpha)^{r}}{r !},
$$

we obtain, for the discrete lattice from Eqs. (22) and (23) in the limit of small excitation number $N$,

$$
\alpha=\frac{\zeta(s)}{8 \zeta(s-2)} N, \quad H=-\frac{\zeta(s)}{64 \zeta(s-2)} N^{3} \text { for } s>3
$$




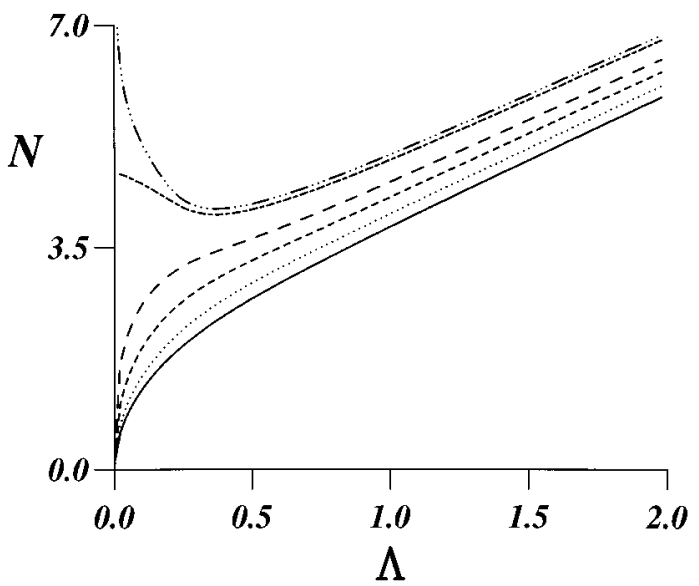

FIG. 4. Number of excitations $N$ versus frequency, $\Lambda$ numerically from Eq. (7) for $s=\infty$ (full line), 4 (dotted line), 3 (shortdashed line), 2.5 (long-dashed line), 2 (short-dash-long-dashed line), and 1.9 (dash-dotted line).

$$
\begin{gathered}
\alpha=\left(\frac{N \zeta(s) \Gamma(s) \sin (\pi s)}{8 \pi(s-1)(s-2)}\right)^{1 /(s-2)}, \\
H=\frac{2-s}{4(s-1)}\left(\frac{\zeta(s) \Gamma(s) \sin (\pi s)}{8 \pi(s-1)(s-2)}\right)^{1 /(s-2)} N^{(2 s-3) /(s-2)}
\end{gathered}
$$

for $s<3$. (27)

The particular value $s=2$ separates two different kinds of behavior: for $s>2, H \rightarrow 0$, while for $1<s<2, H \rightarrow \infty$ when $N \rightarrow 0$ and the stable continuumlike soliton disappears. This is confirmed by our simulation (see Fig. 2).

\section{B. Intersite localized states: $\delta=1 / 2$}

In Fig. 4 we plot the excitation number $N$ as a function of the frequency $\Lambda$ for intersite localized states obtained from the numerical solution of Eq. (8). The analytical results obtained from Eqs. (22) and (23) for $\sigma=1$ and $\delta=1 / 2$ are in qualitative agreement with the numerical results, but the quantitative agreement is not as good as in the on-site soliton case. It is seen from Fig. 4 that this dependence is similar to the dependence $N(\Lambda)$ obtained for on-site localized states (see Figs. 1 and 2) but with the following distinctions.

(i) While the $N(\Lambda)$ curves for on-site states with different dispersion parameters $s$ tend to coincide at high $\Lambda$, the same curves for intersite states become parallel at $\Lambda \rightarrow \infty$. The reason for this difference is seen from the definition of the frequency given by Eqs. (19), (20), and (23): for $\delta=1 / 2$

$$
N=2 \Lambda+4-\frac{2}{\zeta(s)} \quad(\Lambda \rightarrow \infty),
$$

while for $\delta=0$

$$
N=\Lambda+2 \quad(\Lambda \rightarrow \infty) .
$$

(ii) The critical value of the dispersion parameter $s_{\mathrm{cr}}^{\prime} \simeq 2.1$ is much less than the value obtained for on-site localized states.

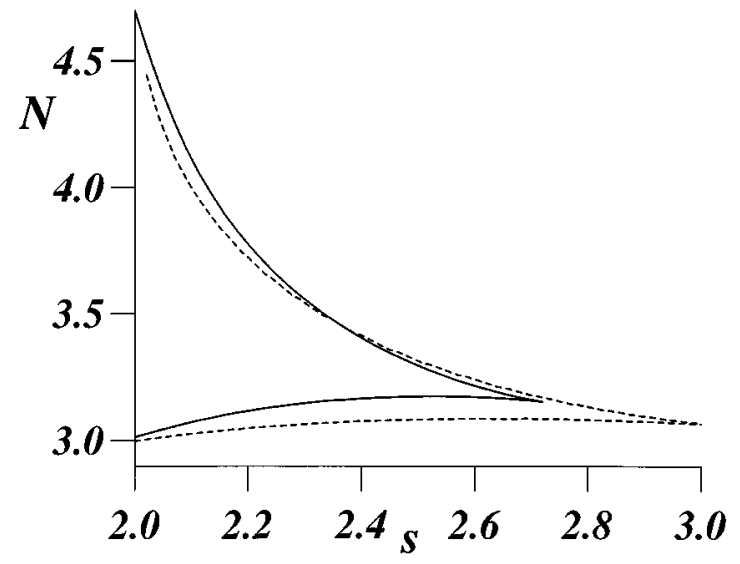

FIG. 5. End points of the bistability interval for $N$ versus the dispersion parameter $s$. For $s=s_{\mathrm{cr}}$ the end points coalesce. Analytical dependence (dashed line) $s_{\mathrm{cr}} \simeq 2.72$ and numerical dependence (full line) $s_{\mathrm{cr}} \simeq 3.03$.

(iii) The interval of $s$ where the two stable intersite states can exist, $2<s<s_{\mathrm{cr}}^{\prime}$, is very narrow. Thus intersite localized states are much less sensitive to the long-range character of the dispersion than the on-site states.

To investigate the stability properties of the different stationary states we use the approach developed in [20] and find that the positive definiteness of the dispersion term $T$ given by Eq. (2) and the form of the nonlinear term $U$ permit generalization of the theorem given by Laedke, Spatschek, and Turitsyn [20] to this nonlocal case. According to this theorem, the necessary and sufficient stability criterion for on-site stationary states is

$$
\frac{d N}{d \Lambda}=\frac{d}{d \Lambda} \sum_{n} \phi_{n}^{2}>0 .
$$

Therefore, we can conclude that in the interval $\left[N_{l}(s), N_{u}(s)\right]$ there are only two linearly stable stationary states $\left[\Lambda_{1}(N)\right.$ and $\left.\Lambda_{3}(N)\right]$. The third state is unstable since $d N / d \Lambda<0$ at $\Lambda=\Lambda_{2}$.

At the points $\Lambda\left(N_{l}\right)$ and $\Lambda\left(N_{u}\right)$ the stability condition is violated since $(\partial N / \partial \Lambda)_{s}$ vanishes. Constructing the locus of the end points, we obtain the curve that is presented in Fig. 5. This curve bounds the region of bistability. It is analogous to the critical curve in van der Waals' theory of liquid-vapor phase transition [37]. Thus, in the present case we have a similar phase transitionlike behavior where the two phases are the continuum states and the intrinsically localized states, respectively. The analog of temperature is the parameter $s$. For the parity-conserving (even) perturbations the stability condition of intersite stationary states is the same as Eq. (30), but these excitations are unstable with respect to paritynonconserving perturbations. A typical evolution of these excitations is presented in Fig. 6. It is seen that by choosing as an initial condition the intersite state it transforms into an intrinsically localized on-site state with a time-dependent width.

Now we turn to discuss stationary states of the discrete NLS model given by Eq. (4) with an arbitrary degree of nonlinearity. The main properties of the system remain un- 

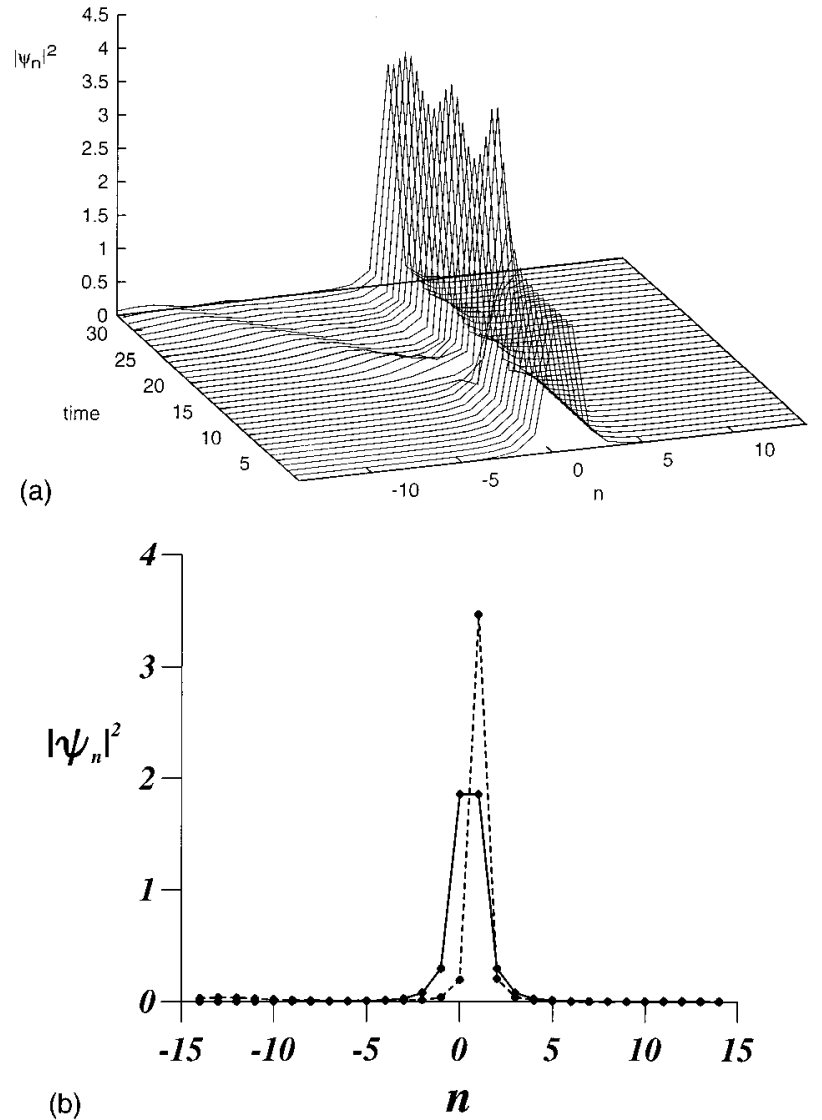

FIG. 6. (a) Evolution of an intersite state $s=2.57, N=4.53$. (b) The intersite state used as the initial condition and the resulting on-site state at $t=40$ where $N=3.99$.

changed, but the critical value of the dispersion parameter $s_{\text {cr }}$ is now a function of $\sigma$. The results of the analytical consideration confirmed by simulation (see Fig. 7) show that $s_{\text {cr }}$ increases when $\sigma$ increases. In particular, for $\sigma \geqslant 1.4$ (the value at which the discrete symmetric ground state can be unstable in the nearest-neighbor approximation [20]; Malomed and Weinstein [34] found the critical value to $\sigma \simeq 1.32$ due to the approximative character of the variational

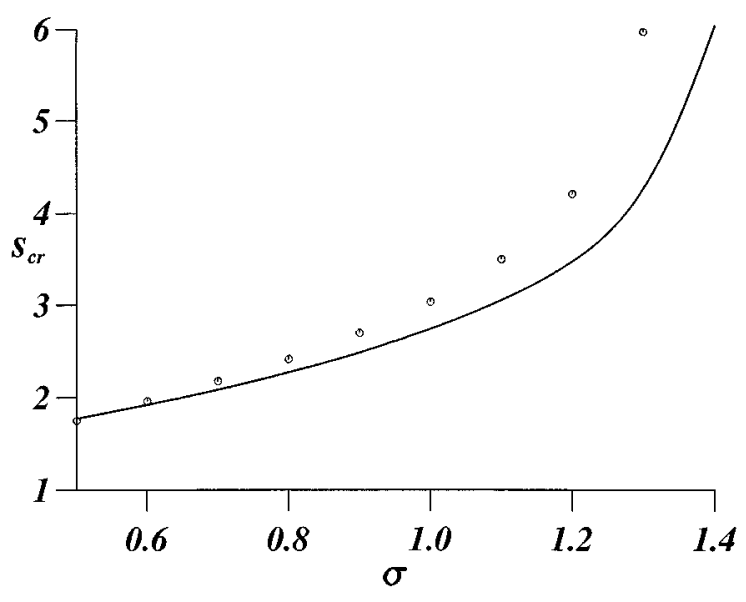

FIG. 7. Critical dispersion parameter $s_{\text {cr }}$ versus the degree of nonlinearity $\sigma$ : analytical dependence (full line) and numerical dependence (circles). approach) the bistability in the nonlinear energy spectrum occurs even for $s \leqslant 6$.

\section{TAILS OF INTRINSICALLY LOCALIZED STATES}

Investigating the asymptotic behavior of the excitations, it is convenient to rewrite Eq. (8) (we consider here the case $\sigma=1$ ) in the form

$$
\phi_{n}=\sum_{m} G_{n-m}(\Lambda) \phi_{n}^{3}
$$

where

$$
G_{n}(\Lambda)=\frac{1}{2 \pi} \int_{-\pi}^{\pi} d k \frac{\cos (k n)}{\Lambda+\mathcal{L}(k)}
$$

is the Green's function. For large $|n|$, the main contribution to the integral on the left-hand side of Eq. (32) is due to small $k$. Hence we can extend the integration over the whole axis and taking into account properties of the Jonqière function [36] in the $k \rightarrow 0$ limit, write the Green's function (32) for $|n| \rightarrow \infty$ as

$$
G_{n}(\Lambda)=\frac{1}{2 \pi} \int_{-\infty}^{\infty} d k \frac{\cos (k n)}{\Lambda+a_{s} k^{\nu(s)}}
$$

where

$$
\nu(s)=\left\{\begin{array}{l}
2 \text { for } s>3 \\
s-1 \text { for } 2<s<3
\end{array}\right.
$$

and

$$
a_{s}= \begin{cases}\frac{\zeta(s-2)}{2 \zeta(s)} \text { for } s>3 & \pi \\ -\frac{\pi}{\Gamma(s) \zeta(s) \cos (\pi s / 2)} & \text { for } 2<s<3 .\end{cases}
$$

Applying Jordan's lemma we get

$$
\begin{gathered}
G_{n}(\Lambda)=\frac{1}{2 \sqrt{\Lambda a_{s}}} e^{-\sqrt{\Lambda / a_{s}}|n|}, \quad s>3 \\
G_{n}(\Lambda)=\frac{1}{\Lambda^{2}}|n|^{-s}, \quad 2<s<3,
\end{gathered}
$$

so the Green's function (32) decays exponentially only for $s>3$. For $s<3$ the exponential decay is replaced by an algebraic decay.

From Eqs. (31), (34), and (35) we see that the leading term in the asymptotic expansion of the excitation wave function $\phi_{n}$ in $|n|$ is given by

$$
\phi_{n} \sim A(\Lambda) G_{n}(\Lambda),
$$

with

$$
A(\Lambda)=\sum_{n} \phi_{n}^{3}
$$




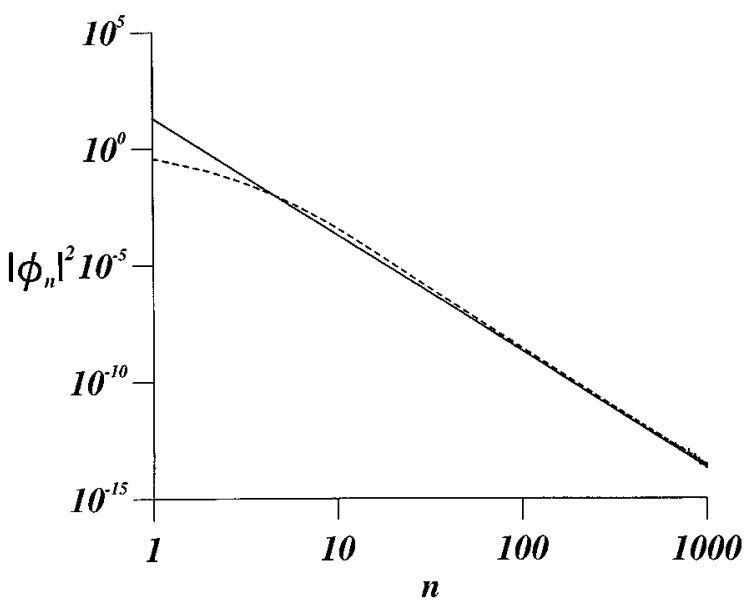

FIG. 8. Shape of the stationary state (dashed line) for $s=2.5$ and $\Lambda=0.7$ and the result of Eq. (41) (full line) on a log-log scale.

It is seen from Eqs. (34)-(36) that for all $s>2, \phi_{n}^{3}$ is a rapidly decaying function of $n$. Hence, being interested in the long-distance behavior of the intrinsically localized states with large frequencies $\Lambda$, we obtain from Eq. (31)

$$
\begin{aligned}
& \phi_{0} \simeq G_{0} \phi_{0}^{3}+2 G_{1} \phi_{1}^{3}, \\
& \phi_{1} \simeq G_{1} \phi_{0}^{3}+2 G_{0} \phi_{1}^{3},
\end{aligned}
$$

where

$$
\begin{gathered}
G_{0}=\frac{1}{\Lambda+1}+O\left(\frac{1}{(\Lambda+1)^{3}}\right), \\
G_{1}=\frac{1}{2(\Lambda+1)^{2}}+O\left(\frac{1}{(\Lambda+1)^{3}}\right) .
\end{gathered}
$$

Using Eqs. (38) and (39), we then get

$$
\begin{gathered}
\phi_{0} \simeq \sqrt{\Lambda+1}, \\
\phi_{1} \simeq \frac{J}{2} \sqrt{\frac{1}{\Lambda+1}} .
\end{gathered}
$$

Inserting Eqs. (17) and (40) into Eq. (36), we obtain that the tails of intrinsically localized states are given by the expressions

$$
\begin{gathered}
\phi_{n} \rightarrow \sqrt{\frac{(\Lambda+1)^{3} \zeta(s)}{2 \Lambda \zeta(s-2)}} \exp \left(-\sqrt{\frac{2 \Lambda \zeta(s)}{\zeta(s-2)}}|n|\right), \quad s>3 \\
\phi_{n} \rightarrow \frac{(\Lambda+1)^{3 / 2}}{\Lambda^{2}}|n|^{-s}, \quad 2<s<3
\end{gathered}
$$

for $|n| \rightarrow \infty$. Thus we can conclude here that only in the case of the short-range dispersion $(s>3)$ do the tails of intrinsically localized states have a usual exponential form. In the systems with long-range dispersive interactions these states have algebraic tails. Figure 8 shows the long-distance behavior of intrinsically localized states for different values of the dispersive parameter $s$. It is seen that the form of the tails

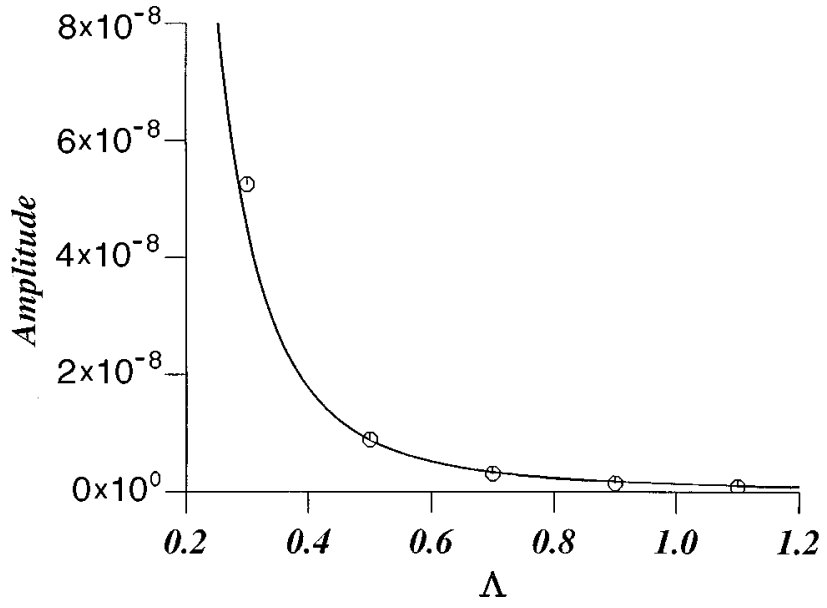

FIG. 9. Amplitude in the tail of the stationary state for $s=2.5$ and $n=450$. Numerical results (circles) and Eq. (41) (full line).

predicted by Eq. (42) is in good agreement with the results of numerical simulations. Moreover, Fig. 9 shows that the $\Lambda$ dependence predicted by Eq. (42) agrees rather well with the numerical results.

\section{QUASICONTINUUM APPROXIMATION}

Now we turn to discuss the quasicontinuum limit of the discrete NLS model given by Eqs. (1)-(3) with arbitrary dispersion parameter $s$ and arbitrary degree of nonlinearity $\sigma$. We are here interested in the case where the characteristic size of the excitations is much bigger than the lattice spacing (which we choose to be equal to unity). It permits us to replace $\psi_{n}(t)$ by the function $\psi(x, t)$ of the continuous variable $x$ and using the Euler-Maclaurin summation formula [38] to obtain instead of Eq. (3) the expression for the potential self-trapping energy

$$
U_{\sigma}=-\frac{1}{\sigma+1} \int_{-\infty}^{\infty} d x|\psi(x, t)|^{2(\sigma+1)}
$$

Under the above-mentioned assumption the most important role in the expression (12) for the kinetic energy $T$ is played by components with small wave numbers $(k \ll 1)$. Therefore, we can safely extend the integration interval to the whole $k$ axis and get

$$
\begin{aligned}
T & =\frac{1}{2 \pi} \int_{-\infty}^{\infty} d k \mathcal{L}(k)|\Psi(k, t)|^{2} \\
& =\int_{-\infty}^{\infty} d x \int_{-\infty}^{\infty} d y\left[\partial_{x} \psi^{*}(x, t)\right] q(x-y) \partial_{y} \psi(y, t),
\end{aligned}
$$

where $\partial_{x} \equiv \partial / \partial x$ and the Fourier transform $Q(k)$ of the kernel $q(x)$ is given by

$$
Q(k)=\frac{1}{k^{2}} \mathcal{L}(k) .
$$

From Eqs. (43) and (44) we obtain that the dynamics of the excitation is governed by the integro-differential NLS equation 


$$
i \partial_{t} \psi+\partial_{x}\left(\hat{q} \partial_{x} \psi\right)+|\psi|^{2 \sigma} \psi=0
$$

where $\hat{q}$ is the Fourier multiplier operator defined by

$$
(\hat{q} \psi)(k)=Q(k) \Psi(k) .
$$

The kernel $q(x)$ in Eqs. (44) and (46) can be considered as a generalized inverse effective mass of excitation. From Eqs. (13) and (45) we obtain that its Fourier transform can be represented in the form

$$
Q(k)=\frac{2}{k^{2}} \operatorname{Re}\left(1-\frac{F\left(e^{i k}, s\right)}{\zeta(s)}\right),
$$

where the Jonqière function $F(z, s)$ is given by Eq. (17). Applying Eq. (25) for the Jonqière function, we obtain in the long-wavelength limit (that is, for $k \ll 1$ ) that the inverse mass function $Q(k)$ can be expressed as

$$
\begin{gathered}
Q(k)=\frac{\zeta(s-2)}{2 \zeta(s)}+O\left(k^{s-3}\right), \quad s>3 \\
Q(k)=\frac{1}{2 \zeta(3)}\left[3+\ln \left(\frac{1}{k^{2}}\right)\right]+O\left(k^{2}\right), \quad s=3,
\end{gathered}
$$

and finally, for $2 \leqslant s<3$,

$$
Q(k)=-\frac{\pi}{\Gamma(s) \zeta(s) \cos \left(\frac{\pi s}{2}\right)}|k|^{s-3}+O\left(k^{0}\right) .
$$

It is seen from Eq. (49) that for $s>3$ the effective mass is constant and Eq. (46) takes a common form characteristic of the NLS models with a short-range dispersive interaction:

$$
i \frac{\partial \psi}{\partial t}+\frac{1}{2 m} \frac{\partial^{2}}{\partial x^{2}} \psi+|\psi|^{2 \sigma} \psi=0,
$$

where $m=\zeta(s) / \zeta(s-2)$. When $s=3$ the dipole-dipole dispersive interaction makes the mass nonlocal, but this nonlocality is rather weak since the inverse-mass function $Q(k)$ given by Eq. (50) is a smooth (logarithmic) function of $k$. In contrast to this, for $2 \leqslant s<3$ the long-range effects make the dispersion essentially nonlocal and, e.g., in the limiting case $s=2$ when, according to Eq. $(51), \mathcal{L} \simeq(6 / \pi)|k|$ the integrodifferential equation (46) can be rewritten as

$$
i \frac{\partial \psi}{\partial t}+\frac{6}{\pi} \mathcal{H}\left\{\frac{\partial \psi}{\partial x}\right\}+|\psi|^{2 \sigma} \psi=0,
$$

where the notation $\mathcal{H}\{f(x)\}$ denotes the Hilbert transform of $f(x)$ given by

$$
\mathcal{H}\{f(x)\} \equiv \mathrm{P} \int_{-\infty}^{\infty} d y \frac{f(y)}{y-x},
$$

where the integral is a Cauchy principal value. Thus the dynamics of self-interacting particles in the systems where the dispersive interaction decrease as $1 / r^{2}$ is governed by Eq. (53), which can be called a NLS-Hilbert equation. At this point it is worth mentioning the formal similarity of Eq. (54) and the perturbed NLS equation that arises from the description of Landau damping in a plasma (see, e.g., Ref. [39]).

Now we want to discuss the stability of the ground states of Eq. (46) with the inverse mass given by Eq. (51). The stationary solutions in the form

$$
\psi(x, t)=\phi(x) e^{i \Lambda t}
$$

of Eq. (46) are stationary points of the Hamiltonian $H=T+U_{\sigma}$ for the fixed excitation number $N$,

$$
\delta_{\phi}(H-\Lambda N)=0
$$

where $\delta_{\phi}$ denotes functional variation with respect to $\phi$ and

$$
N=\int_{-\infty}^{\infty} d x|\psi(x, t)|^{2}
$$

is the continuum analog of Eq. (5). Let $\phi(x, \Lambda)$ be a solution of Eq. (56) and use the scaling transformation

$$
\phi_{p}(x, \Lambda)=p^{1 / 2} \phi(p x, \Lambda)
$$

with the scaling factor $p$. The transformation (58) conserves $N$. For this transformation the functional $H$ becomes a function of the parameter $p$, so that the functional variation changes into a variation of $p$. The function $H(p)$ has an extremum at $p=1$ and we must determine whether it corresponds to a maximum or a minimum. Inserting the function (58) into Eqs. (43) and (48) we get

$$
\begin{gathered}
U_{\sigma}(p) \equiv-\frac{1}{\sigma+1} \int_{-\infty}^{\infty}\left|\phi_{p}(x, \Lambda)\right|^{2(\sigma+1)} d x=p^{\sigma} U_{\sigma}(p=1) \\
T(p) \equiv \int_{-\infty}^{\infty} d x \int_{-\infty}^{\infty} d y\left[\partial_{x} \phi_{p}(x)\right] q(x-y) \partial_{y} \phi_{p}(y) \\
=p^{s-1} T(p=1) .
\end{gathered}
$$

Note that $(s-1) T(p=1)=-\sigma U_{\sigma}(p=1)$ since $d H(p) /$ $\left.d p\right|_{p=1}=0$ and

$$
\left.\frac{d^{2} H(p)}{d p^{2}}\right|_{p=1}=(s-1)(s-\sigma-1) T(p=1) .
$$

Since the quantity $T(p=1)$ is always positive, we may conclude from Eq. (61) that for

$$
s<\sigma+1
$$

the function $H(p)$ at $p=1$ has a maximum and the ground state $\phi(x, \Lambda)$ is unstable. In particular, the continuum NLS models with the degree of nonlinearity $\sigma=1$ and dispersive interactions that decrease slower than $1 / r^{2}$ have unstable ground states.

As it was discussed above, the ground state of the NLS model with the degree of nonlinearity $\sigma \geqslant 1$ and $s=2$ is unstable, while it is stable when $\sigma<1$ [40]. Therefore, we may conclude that for the degree of nonlinearity $\sigma=1$ the lowfrequency (continuumlike) stationary states of the NLS models are exponential-like for $s>2$ and only for $s<2$ they have algebraic tails (see Sec. II). 


\section{CONCLUSION}

In summary, we have proposed a nonlocal discrete nonlinear Schrödinger model for self-interacting excitations with power dependence on the distance $r^{-s}$ of the matrix element of the excitation transfer. We have shown that the behavior of all NLS models with a dispersion interaction decreasing faster than $r^{-s_{\text {cr }}}$ is qualitatively the same as the NLS model with a nearest-neighbor excitation transfer. In contrast to this there is an excitation number interval of bistability in the NLS models with a long-range dispersive interaction $s<s_{\text {cr }}$. In this interval two stable stationary states exist at each excitation number $N$. One of these states is a continuumlike soliton and the other one is an intrinsically localized mode. The existence of the bistability phenomenon in the NLS models with a nonlocal dispersion is a result of the competition of two length scales that exist in the system: the scale related to the competition between nonlinearity and dispersion, and the scale related to the dispersion interaction.

We have considered two types of stationary states: on-site and intersite states. We found that the critical value of the dispersion parameter $s_{\mathrm{cr}}$ for the intersite standing state is slightly above 2 , while for the on-site stationary state it exceeds 3 . This means that the bistable behavior may occur in the case of self-interacting excitations with the dipole-dipole excitation transfer.

We have shown that the long-distance behavior of intrinsically localized states in discrete NLS models with a nonlo- cal dispersion depends drastically on the value of the dispersive parameter $s$. The excitation wave functions decay exponentially only for short-range dispersions. The nonlinear excitations have algebraic tails in the systems where the matrix element of excitation transfer depends on the distance slower than $1 / r^{3}$.

We have also presented the quasicontinuum version of our model. The dynamics of self-interacting excitations in the continuum approximation is described by a nonlocal NLS equation. In the case of the inverse square dependence $\left(r^{-2}\right)$ of the matrix element of the excitation transfer this equation reduces to an equation that is a close analog of the Benjamin-Ono equation in the theory of deep water waves and may be referred to as the Hilbert-NLS equation. Finally, the stability condition for the ground state of this equation was derived.

\section{ACKNOWLEDGMENTS}

We thank J. Juul Rasmussen, V. Mezentsev, and M. Johansson for helpful discussions. Yu.B.G. would like to express his thanks to the Department of Mathematical Modelling and MIDIT, Technical University of Denmark where part of this work was done. Financial support from The Danish Natural Research Foundation (Grant No. 11-0921-1) and from The Ukrainian Fundamental Research Foundation (Grant No. 2.4/355) is also acknowledged.
[1] A. S. Davydov, Soliton in Molecular Systems (Reidel, Dordrecht, 1985).

[2] A. C. Scott, Phys. Rep. 217, 1 (1992).

[3] W. P. Su, J. R. Schrieffer, and A. J. Heeger, Phys. Rev. B 22, 2099 (1980).

[4] A. R. Bishop et al., Phys. Rev. Lett. 52, 671 (1984).

[5] M. J. Ablowitz and J. F. Ladik, Stud. Appl. Math. 55, 213 (1976).

[6] A. C. Scott and L. MacNeil, Phys. Lett. 98A, 87 (1983).

[7] D. J. Kaup, B. A. Malomed, and R. S. Tasgal, Phys. Rev. E 48, 3049 (1993).

[8] A. J. Sievers and S. Takeno, Phys. Rev. Lett. 61, 970 (1988).

[9] V. M. Burlakov, S. A. Kiselev, and V. N. Pyrkov, Phys. Rev. 42, 4921 (1990).

[10] R. Bourbonnais and R. Maynard, Phys. Rev. Lett. 64, 1397 (1990).

[11] S. R. Bickham et al., Phys. Rev. B 43, 2339 (1991).

[12] J. C. Eilbeck and R. Flesh, Phys. Lett. A 149, 200 (1990).

[13] T. Dauxois, M. Peyrard, and A. R. Bishop, Phys. Rev. E 47, 684 (1993).

[14] R. S. MacKay and S. Aubry, Nonlinearity 7, 1623 (1994).

[15] S. Takeno, J. Phys. Soc. Jpn. 58, 759 (1989).

[16] M. Salerno, Phys. Rev. A 46, 6856 (1992).

[17] D. Cai, A. R. Bishop, and N. Gronbech-Jensen, Phys. Rev. Lett. 72, 591 (1994).

[18] D. Hennig, N. G. Sun, H. Gabriel, and G. P. Tsironis, Phys. Rev. E 52, 255 (1995); D. Hennig, K. Ф. Rasmussen, H. Gabriel, and A. Bülow, ibid. 54, 5788 (1996).
[19] O. Bang, J. Juul Rasmussen, and P. L. Christiansen, Nonlinearity 7, 205 (1994).

[20] E. W. Laedke, K. H. Spatschek, and S. K. Turitsyn, Phys. Rev. Lett. 73, 1055 (1994).

[21] A. S. Davydov, Theory of Molecular Excitons (Plenum, New York, 1971).

[22] Y. Ishimori, Prog. Theor. Phys. 68, 402 (1982).

[23] O. M. Braun, Yu. S. Kivshar, and I. I. Zelenskaya, Phys. Rev. B 41, 7118 (1990).

[24] P. Woafo, J. R. Kenne, and T. C. Kofane, J. Phys. Condens. Matter 5, L123 (1993).

[25] G. A. Baker, Jr., Phys. Rev. 122, 1477 (1961).

[26] A. M. Kac and B. C. Helfand, J. Math. Phys. 4, 1078 (1972).

[27] L. Vazquez, W. A. B. Evans, and G. Rickayzen, Phys. Lett. A 189, 454 (1994).

[28] Yu. Gaididei, N. Flytzanis, A. Neuper, and F. G. Mertens, Phys. Rev. Lett. 75, 2240 (1995).

[29] A. Dikande and T. C. Kofane, Physica D 83, 450 (1995).

[30] G. L. Alfimov, V. M. Eleonskii, N. E. Kulagin, and N. V. Mitskevich, CHAOS 3, 405 (1993).

[31] Yu. B. Gaididei, S. F. Mingaleev, P. L. Christiansen, and K. Ф. Rasmussen, Phys. Lett. A 222, 152 (1996); Yu. B. Gaididei et al., Phys. Scr. T67, 151 (1996).

[32] Yu. B. Gaididei, S. F. Mingaleev, P. L. Christiansen, and K. Ф. Rasmussen (unpublished).

[33] R. Blaha, E. W. Laedke, and K. H. Spatschek, Physica D 40, 249 (1989); E.W. Laedke, R. Blaha, K. H. Spatschek, and E. 
A. Kuznetsov, J. Math. Phys. 33, 967 (1992).

[34] B. Malomed and M. I. Weinstein, Phys. Lett. A 220, 91 (1996).

[35] A. B. Aceves et al., Phys. Rev. E 53, 1172 (1996).

[36] W. Magnus, F. Oberhettinger, and R. P. Soni, Formulas and Theorems for the Special Functions of Mathematical Physics (Springer-Verlag, Berlin, 1966).

[37] L. D. Landau and E. M. Lifshitz, Statistical Physics (Perga- mon, London, 1959).

[38] Handbook of Mathematical Functions, edited by M. Abramowitz and I. Stegun (Dover, New York, 1972).

[39] Yu. S. Kivshar and B. A. Malomed, Rev. Mod. Phys. 61, 763 (1989).

[40] In the particular case $\sigma=1 / 2$ the ground-state solution of Eq. (53) is $\psi(x, t)=(N / 6)\left[1+(\pi N x / 72)^{2}\right]^{-1} e^{i(N t / 3)}$, which, from numerical simulations, appears to be stable. 